\title{
Theoretical and Experimental Investigation into Generation of Wrinkling and Slip in Plastic-Films under Transportation*
}

\author{
Hiromu HASHIMOTO ${ }^{* *}$ \\ **Department of Mechanical Engineering, Tokai University, \\ 1117 Kitakaname, Hiratsuka-shi, Kanagawa, 259-1292, Japan \\ E-mail: hiromu@keyaki.cc.u-tokai.ac.jp
}

\begin{abstract}
In the transport processes of plastic-films, wrinkling is sometimes generated due to misalignment of roller. To avoid the wrinkling, it is effective to reduce the web tension. However, under the low web tension, the traction inevitably falls as transport velocity increases due to air entrainment and is accompanied by the onset of slip. This paper examined such a trade-off relation between the wrinkling generation due to roller misalignment and onset of slip. At first, the prediction models for slippage and wrinkling are formulated based on the contact mechanics. Moreover, the observation method of wrinkling generation and slip was presented and the critical misalignment angle, critical web tension to generate wrinkling and slip onset velocity were measured for various operation conditions. Five types of PET (polyethylene terephthalate) films were used for measurements. From the experimental and theoretical results, the effects of roller velocity, anisotropic Young's modulii and film thickness on the relation between the roller misalignment and web tension were made clear. As a summary of experimental and theoretical works, the stable transportation map was newly introduced for protecting both wrinkling and slip.
\end{abstract}

Key words: Web Handling, Traction, Plastic-Film, Slip, Wrinkling, Contact Mechanics, Modeling, Experiment

\section{Introduction}

In recent years, the commercial market of flat panel display such as liquid crystal film is increasingly expanded, and the improvement of the quality of handling system for the production of continuous thin flexible films (hereafter called web) is strongly required. Especially, to establish the web handling technology for preventing the serious web defects such as scratches due to the slippage between the web and roller and wrinkling of webs is the most important engineering problems to be solved quickly in the web handling industries.

In the web transport processes, the webs are driven by the traction generated at the interface between the web and roller. The traction inevitably falls as transport velocity increases due to an air entrainment and is accompanied by the onset of slippage. As a result, the traction limits the productivity of webs. Therefore, an understanding of traction between the web and roller and a formulating the prediction model of slip onset condition, are clearly desirable. At the same time, when the web wrinkles are generated in the handling processes, the wrinkles immediately result in quality loss, so it is also very important to predict and prevent the wrinkles.

${ }^{*}$ Received 30 July, 2009 (No. 09-0396) [DOI: 10.1299/jamdsm.4.238]

Copyright $@ 2010$ by JSME 
On the modeling of traction between the web and roller, Good et.al ${ }^{(1)}$ obtained the empirical formula to express the effective friction coefficient which covers the boundary, mixed and fluid film lubrication regimes. Based on the formula mentioned above, Hashimoto et.al $^{(2)}$ proposed the theoretical model to predict the slip onset velocity and compared the predicted results by the model with the measured data. On the other hand, Good et.al ${ }^{(1)}$ proposed the theoretical model for predicting the generation of wrinkling of isotropic web based on the linearized buckling theory. Moreover, Hashimoto ${ }^{(3)}$ extended the Good's model to anisotropic webs like papers and compared the predicted results with the measured data. In these papers, the empirical formula for the effective friction coefficient mentioned above was used. However, this model of effective friction coefficient is not an essential because it was derived by approximating directly the empirical data of friction coefficient in the mixed lubrication regime as a simple linear function and there was no theoretical background.

In this paper, the prediction model for slippage and wrinkling are newly formulated using the effective friction coefficient based on the contact mechanics between the web and roller, and the predicted results are compared with the measured data to verify the applicability of the model.

\section{Nomenclature}

$A_{a} \quad$ : nominal contact area $\left[\mathrm{m}^{2}\right]$

$A_{f} \quad:$ air film lubrication area $\left[\mathrm{m}^{2}\right]$

$a \quad$ : span between rollers [m]

$E \quad$ : equivalent Young's modulus of asperities [GPa]

$E_{x}, E_{z}$ : Young's modulus of web in the machine and cross machine directions [GPa]

$F \quad:$ friction force or traction transmitted between web and roller [N]

$F_{c} \quad$ : Coulomb friction force $[\mathrm{N}]$

$F_{f} \quad$ : viscous friction force [N]

$f \quad$ : bearing resistance $[\mathrm{N}]$

$h \quad:$ air film thickness $[\mu \mathrm{m}]$

I : inertia moment of roller $\left[\mathrm{kgm}^{2}\right]$

$N \quad$ : normal load [N]

$N_{c} \quad$ : normal contact force [N]

$N_{f} \quad$ : air film reaction force [N]

$n \quad$ : number of asperities

$R \quad$ : radius of roller [m]

$r \quad$ : equivalent curvature of asperity peaks $[\mu \mathrm{m}]$

$r_{b} \quad$ : radius of bearing [m]

$s \quad:$ slip ratio

$T \quad$ : inlet tension $[\mathrm{N} / \mathrm{m}]$

$T^{\prime} \quad$ : outlet tension $[\mathrm{N} / \mathrm{m}]$

$T_{s p} \quad$ : tension to start slippage $[\mathrm{N} / \mathrm{m}]$

$T_{w k} \quad$ : tension to generate wrinkling $[\mathrm{N} / \mathrm{m}]$

$t_{w} \quad:$ web thickness $[\mu \mathrm{m}]$

$U \quad$ : composite velocity of web and roller $\left(U_{r}+U_{s}\right)[\mathrm{m} / \mathrm{s}]$

$U_{r} \quad:$ roller velocity $[\mathrm{m} / \mathrm{s}]$

$U_{s p} \quad$ : roller velocity to start slippage $[\mathrm{m} / \mathrm{s}]$

$U_{w} \quad:$ web velocity $[\mathrm{m} / \mathrm{s}]$

$W \quad$ : web width $[\mathrm{m}]$

$x \quad$ : coordinate in the machine direction [m]

$z \quad:$ coordinate in the cross machine direction [m]

$\alpha \quad:$ parameter defined in Eq.(13)

$\beta \quad$ : parameter defined in Eq.(21) 
$\gamma \quad$ : parameter defined in Eq.(31)

$\delta \quad:$ parameter defined in Eq.(10)

$\zeta_{1} \quad$ : ratio of Young's moduli defined in Eq.(29)

$\zeta_{2} \quad$ : parameter defined in Eq.(29)

$\eta \quad:$ viscosity of air $[\mathrm{Pa} \cdot \mathrm{s}]$

$\Theta \quad$ : web wrap angle [rad]

$\theta \quad$ : misalignment angle of roller [rad]

$\theta_{w k} \quad$ : critical misalignment angle of roller to generate wrinkling [rad]

$\mu_{c} \quad$ : boundary friction coefficient

$\mu_{e f f} \quad:$ effective friction coefficient

$\mu_{s p} \quad$ : friction coefficient to start slippage

$\mu_{s} \quad$ : static friction coefficient between web and roller

$v_{x}, v_{z}$ : Poisson's ratio in $x$ and $z$ directions

$\sigma \quad$ : composite rms roughness between web and roller $[\mu \mathrm{m}]$

$\sigma_{r} \quad:$ rms roughness of roller surface $[\mu \mathrm{m}]$

$\sigma_{w} \quad:$ rms roughness of web surface $[\mu \mathrm{m}]$

$\sigma_{e} \quad:$ stress defines in Eq.(29) $[\mathrm{Pa}]$

$\sigma_{x} \quad$ : normal stress in the machine direction $\left(=T / t_{w}\right)[\mathrm{Pa}]$

$\sigma_{z c r} \quad$ : critical buckling stress of web between rollers $[\mathrm{Pa}]$

$\dot{\omega} \quad:$ angular acceleration of roller $\left[\mathrm{rad} / \mathrm{s}^{2}\right]$

$\chi_{c}, \chi_{f}:$ parameters defined in Eq. $(4)$

\section{Theoretical Modeling}

\subsection{Effective friction coefficient}

The occurrence of web defects such as slippage and wrinkling strongly depends on the magnitude of friction coefficient between the web and roller. As the web transport velocity increases more and more, the friction coefficient becomes lower and lower due to the effect of air entrainment into the gap between the web and roller, and this type of friction coefficient is called "effective" friction coefficient. According to Good et.al ${ }^{(1)}$, the effective friction coefficient is given by the following empirical formula;

$$
\mu_{\text {eff }}= \begin{cases}\mu_{s} & (h<\sigma) \\ \frac{\mu_{s}}{2}\left(3-\frac{h}{\sigma}\right) & (\sigma \leq h \leq 3 \sigma) \\ 0 & (h>3 \sigma)\end{cases}
$$

where the composite rms roughness $\sigma$ is defined as follows.

$$
\sigma=\sqrt{\sigma_{w}^{2}+\sigma_{r}^{2}}
$$

The effective friction coefficient in Eq. (1), however, was obtained directly from the measured data and there is no guarantee of its theoretical validity. Therefore, in this paper the effective friction coefficient is newly formulated based on the contact mechanics proposed by Greenwood and Williamson ${ }^{(4)}$.

Figure 1 shows the interface between the web and roller. Usually, the friction condition between the moving web and the rotating roller is considered as mixed lubrication condition as shown in Fig.2.

From Fig.2, the force balance equations in the normal and tangential directions are given, respectively, as follows;

$$
N=N_{c}+N_{f}, F=F_{c}+F_{f}
$$

Dividing Eq.(3) by the total normal force $N$, the following equations are obtained; 


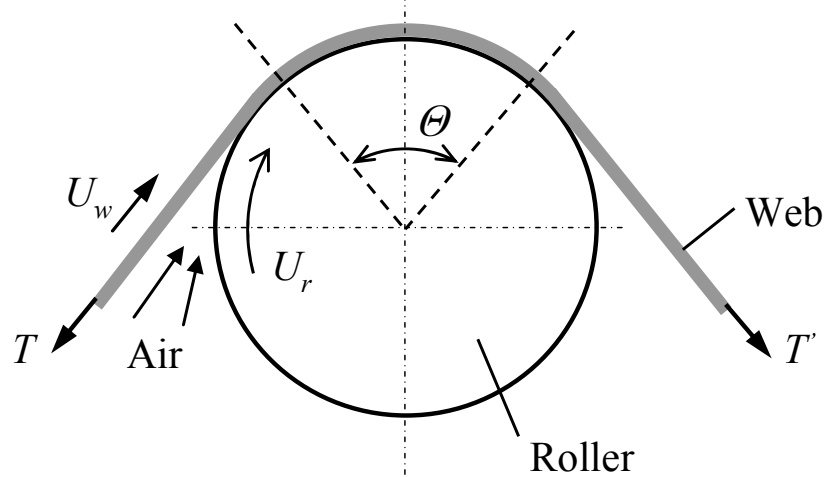

Fig.1 Interface between web and roller

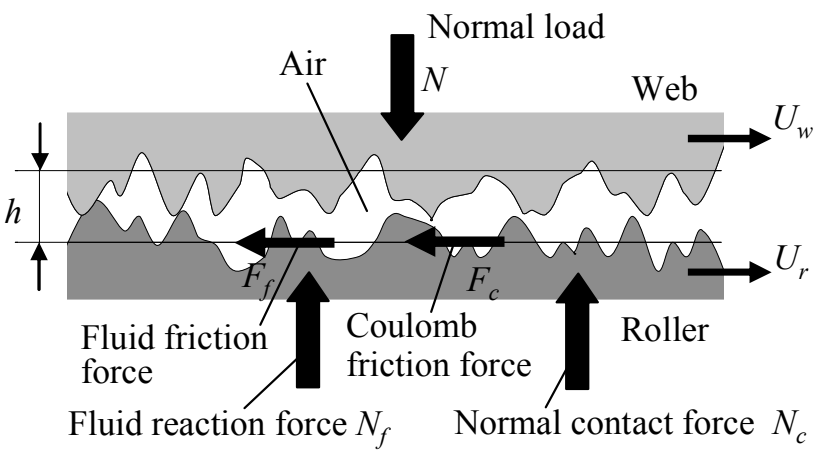

Fig. 2 Mixed lubrication between web and roller

$$
\begin{aligned}
& \frac{N_{c}}{N}+\frac{N_{f}}{N}=\chi_{c}+\chi_{f}=1 \\
& \mu_{\text {eff }}=\frac{F_{c}}{N_{c}} \frac{N_{c}}{N}+\frac{F_{f}}{N_{f}} \frac{N_{f}}{N}=\mu_{c} \chi_{c}+\mu_{f} \chi_{f}
\end{aligned}
$$

where the friction coefficients $\mu_{\text {eff }}$ and $\mu_{c}$ are defined, respectively, by;

$$
\mu_{\text {eff }}=\frac{F}{N}, \mu_{c}=\frac{F_{c}}{N_{c}} \cong \mu_{s}
$$

and the friction coefficient $\mu_{f}$ is determined by the fluid film lubrication theory as follows.

$$
\mu_{f}=\frac{F_{f}}{N_{f}} \cong \frac{\eta\left|U_{r}-U_{W}\right| A_{f}}{(T / R) h A_{f}}=\frac{\eta R\left|U_{r}-U_{W}\right|}{h T}
$$

Usually, the web is transported under the condition of $U_{r}=U_{w}$, and then from Eq.(4) through Eq.(7), the effective friction coefficient is expressed as follows.

$$
\mu_{\text {eff }}=\mu_{s} \chi_{c}
$$

Following the same manner proposed by Greenwood and Williamson ${ }^{(4)}$, assuming that the probability density of asperity height of surface roughness follows the Gaussian distribution and the geometry of asperity can be treated as a semi-sphere as shown in Fig.3, the normal contact force $N_{c}$ is determined as follows;

$$
\begin{aligned}
N_{c} & =\frac{2}{3} n E r^{1 / 2} \int_{h}^{\infty}(\xi-h)^{3 / 2} \frac{1}{\sqrt{2 \pi} \sigma} e^{-\xi^{2} / 2 \sigma^{2}} d \xi \\
& \cong \frac{2}{3} n E r^{1 / 2} \int_{h}^{\infty}(\xi-h)^{3 / 2} \frac{1}{\sigma} e^{-\xi / \sigma} d \xi \\
& =\frac{\sqrt{\pi}}{2}(\delta \sigma r) E A_{a}\left(\frac{\sigma}{r}\right)^{1 / 2} e^{-h / \sigma}
\end{aligned}
$$



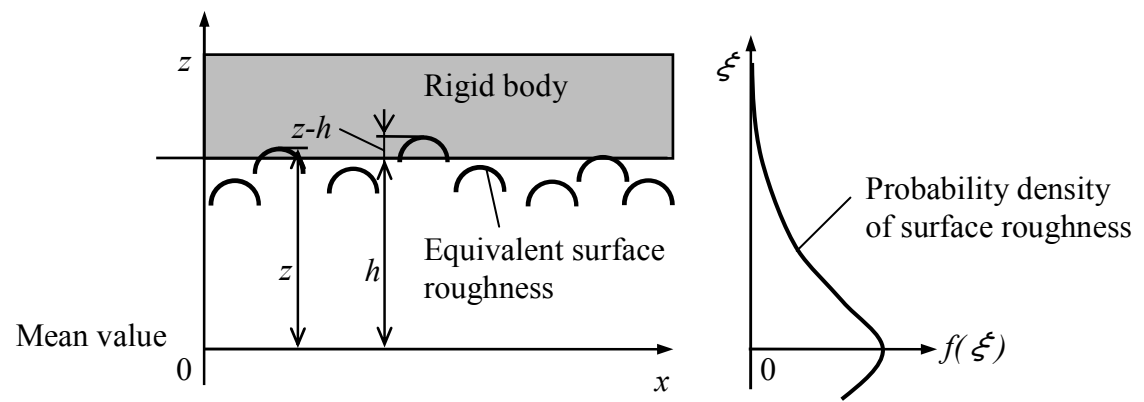

Fig. 3 Contact model between web and roller

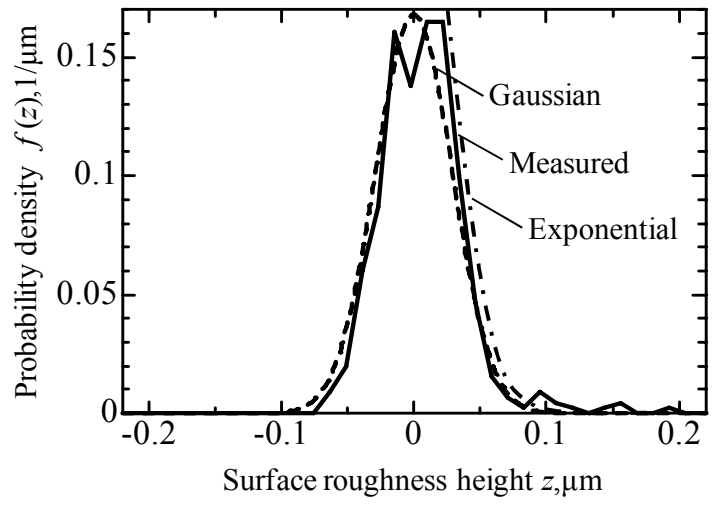

Fig.4 Probability density of surface roughness height for PET film where the parameter $\delta$ is a density of asperities defined as;

$$
\delta=\frac{n}{A_{a}}
$$

Figure 4 shows an example of the measured data on the probability density of surface roughness height for PET film. As can be seen in the figure, the exponential model reasonably agrees with the Gaussian model and measured data.

On the other hand, the total normal force $N$ is expressed as;

$$
N=\frac{T}{R} A_{a}
$$

From Eqs.(9) and (11), the parameter $\chi_{c}$ in Eq.(8) is determined as follows;

$$
\chi_{c}=\frac{N_{c}}{N}=\alpha \frac{R}{T} e^{-h / \sigma}
$$

where the parameter $\alpha$ is defined as ;

$$
\alpha=\frac{\sqrt{\pi}}{2}(\delta \sigma r) E\left(\frac{\sigma}{r}\right)^{1 / 2}
$$

and $E$ and $r$ in Eq.(13) mean the equivalent Young's modulus and curvature of surface asperities of web and roller, respectively.

When $\chi_{c}=1$, the effect of hydrodynamic lubrication will disappear. And then, substituting $\chi_{c}=1$ into Eq.(12), the critical air film thickness changed from mixed lubrication condition to solid contact condition, $h_{c r}$, is obtained as follows.

$$
h_{c r}=\sigma \ln \left(\alpha \frac{R}{T}\right) ; \alpha \frac{R}{T}>1
$$

Summarizing these results, the effective friction coefficient based on the contact 


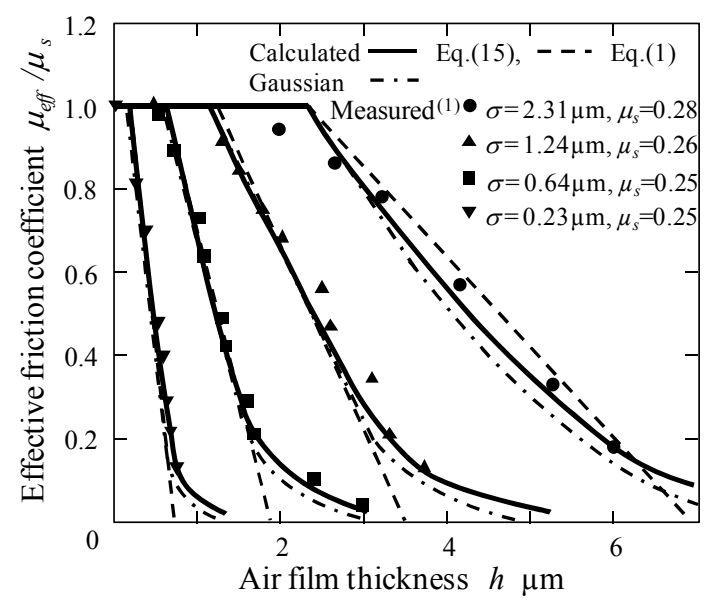

Fig.5 Variation of effective friction coefficient with air film thickness for $R=0.037[\mathrm{~m}], T=200[\mathrm{~N}], t_{w}=35.6[\mu \mathrm{m}], \Theta=90[\mathrm{deg}]$

mechanics is expressed as:

$$
\mu_{\text {eff }}= \begin{cases}\mu_{s} & \left(h<h_{c r}\right) \\ \mu_{s} \alpha \frac{R}{T} e^{-h / \sigma} & \left(h \geq h_{c r}\right)\end{cases}
$$

Newly obtained exponential type of Eq.(15) depends on many parameters such as equivalent Young's modulus $E$, equivalent curvature $r$, density of asperities $\delta$, rms roughness $\sigma$, roller radius $R$, roller velocity $U_{r}$, and web tension $T$.

Figure 5 shows a comparison of calculated results on the effective friction coefficient by Eq.(15) and the measured data ${ }^{(1)}$, in which the calculated results by Eq.(1) and the Gaussian model are also indicated. In Eq.(15), $\delta \sigma r$ and $\sigma / r$ were determined statistically from the measured results of surface roughness profile and then the value of $\alpha$ was obtained as $\alpha \cong 1.51 \times 10^{4}$. The calculated results by Eq.(15) reasonably agree with the results by the Gaussian model and the measured data, and the applicability of Eq.(15) is verified experimentally. On the other hand, the results by Eq.(1) underestimate the effective friction coefficient around $h=3 \sigma$, which may influence on the onset of slippage.

\subsection{Slip onset condition}

From Fig.6, using the Euler's belt theory the traction torque transmitted between the web and roller is given as;

$$
T_{r}=W R\left(T^{\prime}-T\right)=W R T\left(e^{\mu_{e f f} \Theta}-1\right)
$$

On the other hand, the slip onset condition between the web and roller is expressed as;

$$
T_{r}=f r+I \dot{\omega}
$$

Equating Eq.(16) and Eq.(17) and solving with respect to $\mu_{e f f}$, the effective friction coefficient to start slippage, $\mu_{s p}$, is obtained as follows.

$$
\mu_{s p}=\frac{1}{\Theta} \ln \left(1+\frac{I \dot{\omega}+f r}{W R T}\right)
$$

In the actual web handling lines, the resistance torque $I \dot{\omega}+f r$ in Eq.(18) is minimized as much as possible. Therefore, usually the ratio of $I \dot{\omega}+f r$ to $W R T$ in Eq.(18) can be treated as a small quantity much less than the unity. From this fact, the right hand side of Eq.(18) is approximated reasonably as follows.

$$
\mu_{s p} \cong \frac{I \dot{\omega}+f r}{\Theta W R T}
$$




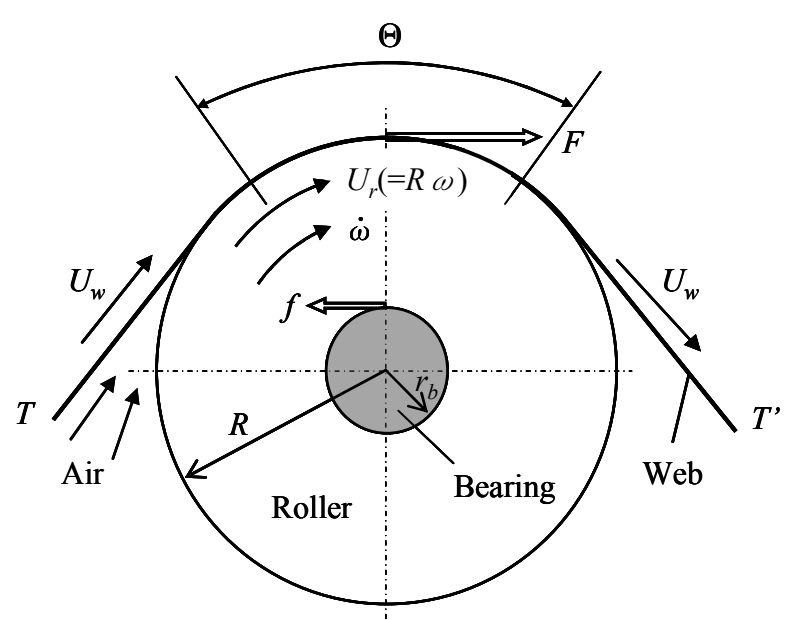

Fig.6 Traction transmitted between web and roller

Equating Eq.(15) and Eq.(19) for $h>h_{c r}$, the critical air film thickness to start slippage, $h_{s p}$, is obtained as;

$$
h_{s p}=\beta \sigma
$$

where the parameter $\beta$ is defined as follows.

$$
\beta=\ln \left(\frac{\alpha \mu_{s} \Theta W R^{2}}{I \dot{\omega}+f r}\right)
$$

According to Hashimoto ${ }^{(5)}$, the averaged air film thickness between the web and roller is given by the following equation.

$$
h=0.589 R\left(\frac{12 \eta U_{r}}{T}\right)^{\frac{2}{3}}
$$

From Eqs.(20) and (22), the slip onset velocity $U_{s p}$ for the fixed tension $T$ is obtained as follows;

$$
U_{s p}=0.184 \beta^{\frac{3}{2}}\left(\frac{\sigma}{R}\right)^{\frac{3}{2}} \frac{T}{\eta}
$$

and the slip onset tension $T_{s p}$ for the fixed velocity $U_{r}$ is also obtained as follows.

$$
T_{s p}=5.422 \beta^{-\frac{3}{2}}\left(\frac{\sigma}{R}\right)^{-\frac{3}{2}} \eta U_{r}
$$

\subsection{Wrinkling onset condition}

According to Hashimoto ${ }^{(3)}$, the wrinkling onset condition for both isotropic and anisotropic webs is given by the following two equations;

$$
\begin{aligned}
& \theta_{w k}=\frac{6 a^{2}}{E_{x} W^{2}} \sqrt{\sigma_{z c r}{ }^{2}-\sigma_{z c r} \sigma_{x}} \\
& T_{w k}=\frac{2 t_{w}{ }^{2}}{\mu_{e f f} W} \sqrt{\frac{E_{x} E_{z}}{3\left(1-v_{x} v_{z}\right)}}
\end{aligned}
$$

where the critical buckling stress $\sigma_{z c r}$ is given as; 


$$
\sigma_{z c r}=\frac{W^{2}}{i^{2} a^{2}}\left\{\sigma_{e}\left\{1+\zeta_{1} i^{4} \frac{a^{4}}{W^{4}}+\zeta_{2} i^{2} \frac{a^{2}}{W^{2}}\right\}-\sigma_{x}\right\}
$$

and the integer $i$ means the wave number to be satisfied with the following inequality;

$$
\sigma_{e}\left\{1-i^{2}(i+1)^{2} \zeta_{1} \frac{a^{4}}{W^{4}}\right\}<\sigma_{x}<\sigma_{e}\left\{1-(i-1)^{2} i^{2} \zeta_{1} \frac{a^{4}}{W^{4}}\right\}
$$

where $\sigma_{x}, \sigma_{e}, \zeta_{1}$ and $\zeta_{2}$ are defined, respectively, as follows.

$$
\begin{aligned}
& \sigma_{x}=\frac{T}{t_{W}}, \sigma_{e}=\frac{\pi^{2} E_{x} t_{W}{ }^{2}}{12 a^{2}\left(1-v_{x} v_{z}\right)}, \\
& \zeta_{1}=\frac{E_{Z}}{E_{x}}, \zeta_{2}=\frac{4\left(1-v_{X} v_{Z}\right)}{1+v_{X}+\left(1+v_{z}\right) / \zeta_{1}}+v_{Z}+v_{X} \zeta_{1}
\end{aligned}
$$

When the misalignment angle of roller, $\theta$, exceeds $\theta_{w k}$ in Eq.(25) and at the same time the web tension, $T$, becomes larger than $T_{w k}$ in Eq.(26), the wrinkles will be generated on the center line of the web wrapped over the roller.

Substituting $\mu_{\text {eff }}$ from Eq.(15) into Eq.(26), the following equation is obtained.

$$
e^{\frac{h}{\sigma}}=T_{w k}\left(\frac{\mu_{s} \alpha W R}{2 t_{w}^{2}} \sqrt{\frac{3\left(1-v_{x} v_{z}\right)}{E_{x} E_{z}}}\right)
$$

Substituting $h$ from Eq.(22) into Eq.(30), the following equation with respect to the wrinkling onset tension $T_{w k}$ is obtained;

$$
T_{w k}=5.422\left(\ln T_{w k}+\gamma\right)^{-\frac{3}{2}}\left(\frac{\sigma}{R}\right)^{-\frac{3}{2}} \eta U_{r}
$$

where the parameter $\gamma$ is defined as follows.

$$
\gamma=\ln \left(\frac{\alpha \mu_{s} W R}{2 t_{w}{ }^{2}} \sqrt{\frac{3\left(1-v_{x} v_{z}\right)}{E_{x} E_{z}}}\right)
$$

Solving Eq.(31) numerically, the wrinkling onset tension $T_{w k}$ is determined.

Summarizing these results, the safety operation region without slippage and wrinkling for the fixed velocity is expressed as follows.

$$
5.422 \beta^{-\frac{3}{2}}\left(\frac{\sigma}{R}\right)^{-\frac{3}{2}} \eta U_{r}<T<T_{w k}
$$

or

$$
\theta \leq \frac{6 a^{2}}{E_{x} W^{2}} \sqrt{\sigma_{z c r}^{2}-\sigma_{z c r} \sigma_{x}} \text { and } T \geq T_{w k}
$$

\section{Experimental Verifications}

To verify the applicability of the prediction model for slippage and wrinkling developed in the former chapter, the slip and wrinkling onset conditions were observed and measured by the experimental apparatus as shown in Fig. 7.

The endless type of test film(7) was supported by the five rollers. The tension was applied by the tension control roller(2), and the magnitude of tension was measured by the load cell(1) and displayed on the tension unit(6). The test roller(3) was driven by the driving motor(8) through a belt. In the experiment of slip onset condition, the velocities of 


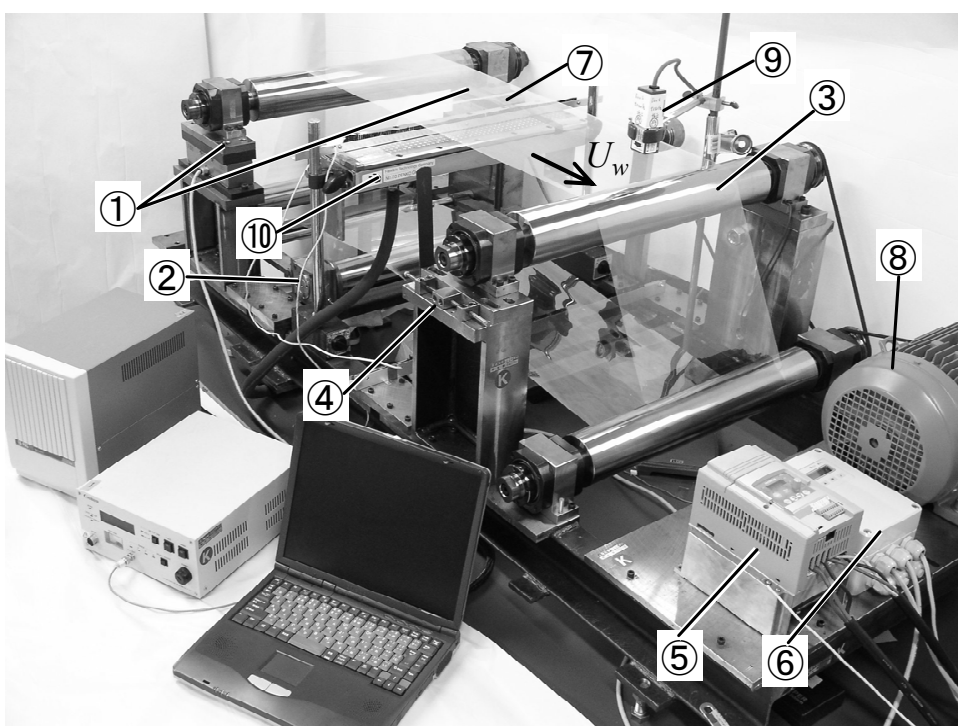

(1) Load cell

(6) Tension unit

(2) Tension control roller

(7) Test film

(3) Test roller

(8) Driving motor

(4) Angle adjustable mechanism

(9) Laser Doppler velocimeter

(5) Speed controller

(10) Air suction device

Fig.7 Experimental apparatus for observing slippage and wrinkling

Table 1 Test conditions

\begin{tabular}{|ll|c|}
\hline \multicolumn{2}{|c|}{ Item } & Value \\
\hline Span & $a, \mathrm{~m}$ & 0.8 \\
\hline Roller radius & $R, \mathrm{~m}$ & $\mathbf{0 . 0 4}$ \\
\hline Web wrap angle & $\Theta, \mathrm{deg}$ & $\mathbf{6 0}$ \\
\hline Web tension & $T, \mathrm{~N} / \mathrm{m}$ & $\mathbf{1 0} \sim 800$ \\
\hline Roller surface velocity & $U_{r}, \mathrm{~m} / \mathrm{s}$ & $\mathbf{0} \sim 0.5$ \\
\hline Roller surface roughness & $\sigma_{r}, \mu \mathrm{m}$ & $\mathbf{0 . 3}$ \\
\hline Web width & $W, \mathrm{~m}$ & 0.3 \\
\hline
\end{tabular}

Table 2 Physical properties of tested plastic-films

\begin{tabular}{|ll|c|c|c|c|c|}
\hline \multicolumn{1}{|c}{ Item } & & $\begin{array}{c}\text { PET } \\
(1)\end{array}$ & $\begin{array}{c}\text { PET } \\
(2)\end{array}$ & $\begin{array}{c}\text { PET } \\
(3)\end{array}$ & $\begin{array}{c}\text { PET } \\
(4)\end{array}$ & $\begin{array}{c}\text { PET } \\
(5)\end{array}$ \\
\hline Young's modulus in MD & $\boldsymbol{E}_{\boldsymbol{y}}, \mathrm{GPa}$ & 5.32 & 4.37 & 3.72 & 1.59 & 4.85 \\
\hline Young's modulus in CD & $\boldsymbol{E}_{z}, \mathrm{GPa}$ & 4.83 & 4.29 & 4.26 & $\mathbf{8 . 7 4}$ & 1.57 \\
\hline Young's modulus ratio & $\zeta_{1}\left(=\boldsymbol{E}_{z} / \boldsymbol{E}_{\boldsymbol{x}}\right)$ & $\mathbf{0 . 9 1}$ & $\mathbf{0 . 9 8}$ & 1.15 & $\mathbf{5 . 5 0}$ & $\mathbf{0 . 3 2}$ \\
\hline Film thickness & $\boldsymbol{t}_{w}, \mu \mathrm{m}$ & 12.0 & $\mathbf{2 5 . 0}$ & $\mathbf{5 0 . 0}$ & $\mathbf{5 0 . 0}$ & $\mathbf{5 0 . 0}$ \\
\hline rms roughness of web & $\sigma_{w}, \mu \mathrm{m}$ & $\mathbf{0 . 0 5 5}$ & $\mathbf{0 . 0 3 2}$ & $\mathbf{0 . 0 3 8}$ & $\mathbf{0 . 0 5 8}$ & $\mathbf{0 . 0 8 2}$ \\
\hline rms composite roughness & $\sigma, \mu \mathrm{m}$ & $\mathbf{0 . 3 0 5}$ & $\mathbf{0 . 3 0 2}$ & $\mathbf{0 . 3 0 2}$ & $\mathbf{0 . 3 0 6}$ & $\mathbf{0 . 3 1 1}$ \\
\hline Static friction coefficient & $\mu_{s}$ & $\mathbf{0 . 3}$ & $\mathbf{0 . 3}$ & $\mathbf{0 . 3}$ & $\mathbf{0 . 2 9}$ & $\mathbf{0 . 2 9}$ \\
\hline
\end{tabular}

transported film and roller were measured by LDV(9) and the slip ratio defined by the flowing equation was immediately calculated.

$$
S=\frac{\left|U_{r}-U_{W}\right|}{U_{r}}
$$

When the slip ratio reached to 0.01 , we have judged the onset of slippage and the roller velocity at that time was recorded as the slip onset velocity $U_{s p}$. 


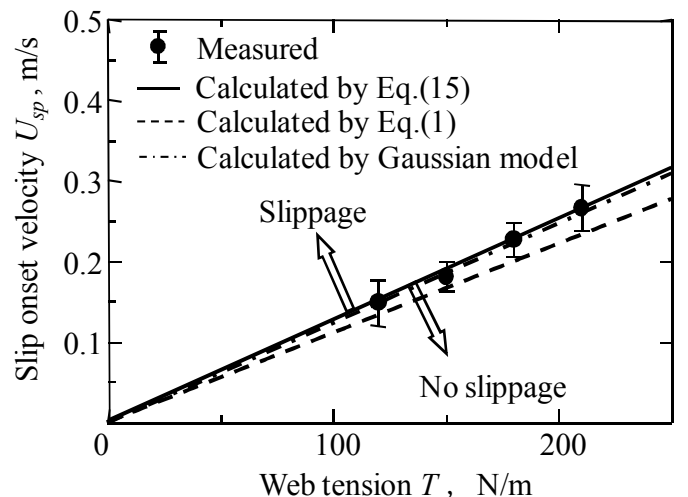

Fig. 8 Variation of slip onset velocity with web tension

On the other hand, in the experiment of wrinkling generation, the misalignment angle of test roller was changed continuously by the angle adjustable mechanism(4) from 0 to 1.5 deg. A procedure for the observation of wrinkles was to fix step by step the web tension for a given velocity and to increase slowly the misalignment angle of roller until the wrinkling was generated completely. The critical angle for generating wrinkling was read through the pitch of angle adjustable mechanism with micro-screw, and recorded by the video-recorder

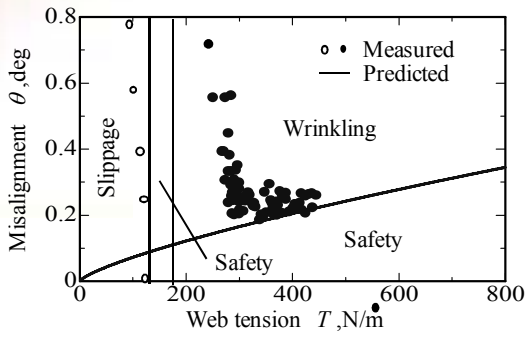

(a) $U_{r}=0.15[\mathrm{~m} / \mathrm{s}]$

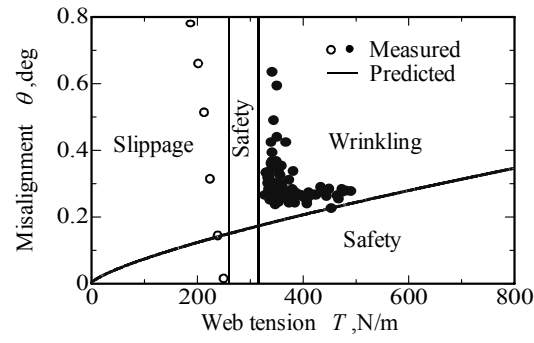

(b) $U_{r}=0.30[\mathrm{~m} / \mathrm{s}]$

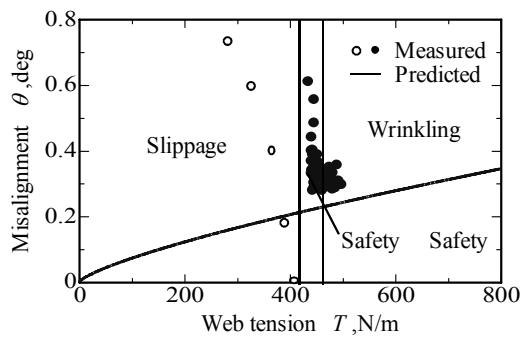

(c) $U_{r}=0.45[\mathrm{~m} / \mathrm{s}]$

( i ) Effect of web velocity on safety region for PET(2)

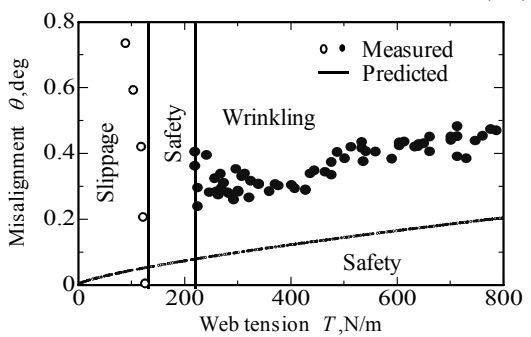

(a) $\zeta_{1}=0.32$ (PET(5))

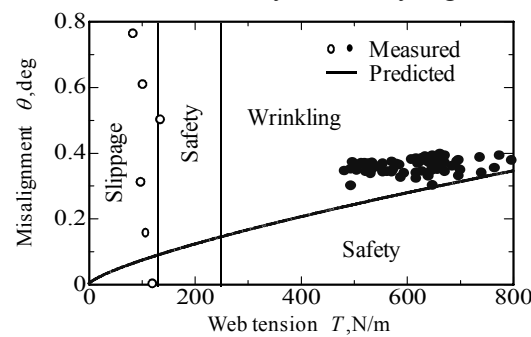

(b) $\zeta_{1}=1.15$ (PET(3)

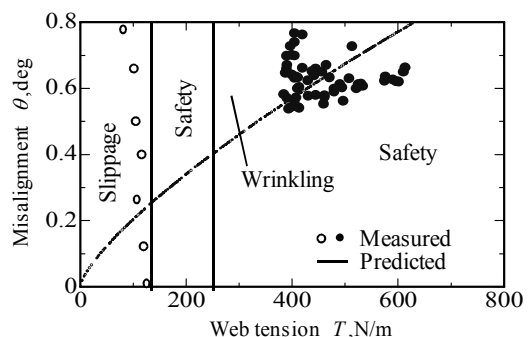

(c) $\zeta_{1}=5.5$ (PET(4)

( ii ) Effect of anisotropic of Young's moduluii on safety region for $U_{r}=0.15[\mathrm{~m} / \mathrm{s}]$

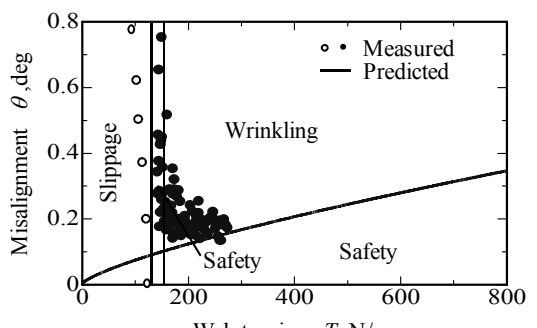

Web tension $T, \mathrm{~N} / \mathrm{m}$ (a) $t_{w}=12[\mathrm{~mm}]$ (PET(1)

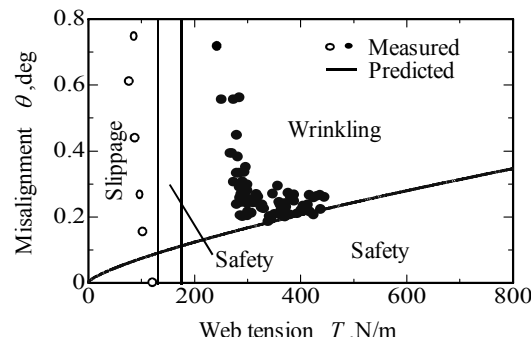

(b) $t_{w}=25[\mathrm{~mm}]$ (PET(2))

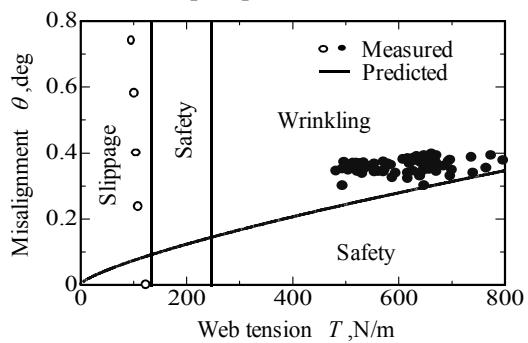

(c) $t_{w}=50[\mathrm{~mm}]$ (PET(3)

(iii) Effect of web thickness on safety region for $U_{r}=0.15[\mathrm{~m} / \mathrm{s}]$

Fig.9 Slippage, wrinkling and safety regions of transported webs 
at the same time. The test conditions are listed in Table 1. In both experiments, five types of PET films as listed in Table 2 were used as test webs, which are manufactured specially for this research work.

Figure 8 shows the variation of slip onset velocity with tension for the test film(3) in Table 2. As can be seen in the figure, the predicted results by Eq.(15) agree well with the measured data and the predicted results by Gaussian model, but the predicted results based on Eq.(1) become smaller about $10 \%$ than the measured data.

Figure 9 shows the predicted results of slippage, wrinkling and safety regions under various test conditions with measured data, in which the white plots mean the onset of slippage and the black plots mean the generation of wrinkling, respectively. The effect of web velocity on the safety region is presented in Fig.9(i). As the web velocity increases, the slip onset tension also increases and the slippage region becomes larger. At that time, the wrinkling onset tension increases considerably and the wrinkling region becomes smaller. The effect of anisotropic Young's modulli on the safety region is shown in Fig.8(ii). As the anisotropic ratio of Young's modulli $\zeta_{1}\left(=E_{z} / E_{x}\right)$ increases, the wrinkling onset tension increases slightly but the slip onset tension is never changed. The critical misalignment angle to generate wrinkling, however, considerably increases with an increase of the anisotropic ratio $\zeta_{1}$. As a result, the safety region without both slippage and wrinkling is remarkably enlarged. The effect of web thickness on the safety region is demonstrated in Fig.9(iii). The slip onset tension is not affected by the web thickness but the wrinkling onset tension becomes larger with an increase of web thickness, and therefore the safety region is enlarged. As can be seen in the figure, the predicted results for both slippage and wrinkling reasonably agree with the measured data, so we can make the stable web transportation map as shown in Fig. 8 by the present model for preventing slippage and wrinkling simultaneously.

\section{Conclusion}

In this paper, the theoretical prediction model for the generation of slippage and wrinkling was newly developed based on the contact mechanics between the web and roller. The predicted results by this model were compared with the measured data under various test conditions and the reasonable agreements were obtained between them. As a summary of the present work, the stable web transportation map for preventing both slippage and wrinkling effectively was introduced.

Finally, the author wishes to acknowledge the financial support of the Ministry of Education, Culture, Sports, Science and Technology of Japan under the Grant in Aid for Science Research (No. 19206021).

\section{References}

(1) Good, J.K., Kedl, D.M. and Shelton, J.J., "Shear Wrinkling in Isolated Spans", Proceedings of the 4th International Conference on Web Handling", Web Handling Research Center, Stillwater, Oklahoma, (1997), pp.462-480.

(2) Hashimoto, H., Ibi, Y., Kiribe, S. and Kondou, C., "Prediction of Slippage Onset Condition Between Web and Steel Roller", Journal of Microsystem Technology, Vol. 13, (2007), pp.965-971.

(3) Hashimoto, H., "Prediction Model of Paper-Web Wrinkling and Some Numerical Calculation Examples with Experimental Verifications", Journal of Microsystem Technology, Vol.13, (2007), pp.933-941.

(4) Greenwood, J.A. and Williamson, J.B.P., "Contact of Nominally Flat Surface", Proceedings of Royal Society of London, Ser.A, Vol.295, (1996), pp.300-319.

(5) Hashimoto, H., "Air Film Thickness Estimation in Web Handling Processes", Journal of Tribology ASME, Vol.121, (1999), pp.50-55. 